Relations industrielles

Industrial Relations

\title{
Standards of Wage Determination, by Paul Bullock, Institute of Industrial Relations, University of California, Los Angeles, 1960, 99 pp. \$0.75.
}

\section{Jean-Paul Deschênes}

Volume 16, numéro 1, janvier 1961

URI : https://id.erudit.org/iderudit/1021898ar

DOI : https://doi.org/10.7202/1021898ar

Aller au sommaire du numéro

Éditeur(s)

Département des relations industrielles de l’Université Laval

ISSN

0034-379X (imprimé)

1703-8138 (numérique)

Découvrir la revue

Citer ce compte rendu

Deschênes, J.-P. (1961). Compte rendu de [Standards of Wage Determination, by Paul Bullock, Institute of Industrial Relations, University of California, Los Angeles, 1960, 99 pp. \$0.75.] Relations industrielles / Industrial Relations, 16(1), 126-127. https://doi.org/10.7202/1021898ar

Tous droits réservés (C Département des relations industrielles de l’Université Laval, 1961
Ce document est protégé par la loi sur le droit d'auteur. L'utilisation des services d'Érudit (y compris la reproduction) est assujettie à sa politique d'utilisation que vous pouvez consulter en ligne.

https://apropos.erudit.org/fr/usagers/politique-dutilisation/ 
économique. Dans les pays dont le développement est lent seul le facteur de refoulement joue. Le revenu agricole est très bas et ne permet pas l'obtention d'un niveau de vie minimum à toute la population rurale. Une partie de cette population est donc refoulée vers les villes. Cependant, l'absence d'emplois non agricoles réduit cette nouvelle population urbaine à un état de misère égal sinon pire que celui qu'elle voulait fuir. Ainsi, dans ces pays, l'émigration rurale ne fait que déplacer les problèmes de la population et peut même nuire au progrès économique si elle est trop considérable.

Sans vouloir infirmer le schéma strictement économique utilisé dans la monographie, nous aimerions suggérer qu'une vue totalement synthétique du problème aurait demandé l'analyse des facteurs sociaux et culturels. En effet, la sociologie rurale, dans le cas des pays évolués, et l'anthropologie, dans le cas des pays dits «sous-développés», montrent bien que la motivation qui pousse le travailleur rural vers la ville n'est pas strictement d'ordre économique.

Ainsi plus que l'écart réel entre revenus urbains et ruraux et que les possibilités réelles d'emploi en dehors de l'agriculture, il semble que ce soit la conscience et la connaíssance par la population rurale de cet écart et de ces responsabilités qui soient les facteurs déterminants. De même, il semble nécessaire que le travailleur rural se juge capable de remplir les exigences du travail urbain avant qu'il n'émigre vers la ville. Ces définitions de la situation par la population rurale présupposent la pénétration dans le milieu rural d'informations et de valeurs nouvelles, c'est-à-dire une certaine acculturation.

De plus, il n'existe pas seulement des facteurs qui favorisent l'émigration rurale. Il existe aussi des facteurs de «freinage ». Notons, entre autres, le système de valeurs et l'organisation sociale qui existent dans le milieu rural (l'opposition à la vie urbaine, la famille patriarcale, etc.). Le travailleur rural en émigrant vers la ville fait plus que changer de lieu de résidence. Très souvent, c'est toute sa culture qu'il doit changer. L'écart entre la culture rurale et la culture urbaine non seulement cause des problèmes d'adaptation mais aussi sert à ralentir le mouvement vers la ville.
La très grande diversité des cultures rend certainement la classification des pays plus difficile que leur diversité plus restreinte sur le plan du développement économique. Même s'il est possible de considérer la situation du Brésil, de l'Irak et de l'Afrique noire comme des situations de développement économique accéléré, il est impossible de considérer la situation culturelle de ces pays comme identique. A notre avis, ce n'est que lorsqu'on pourra tenir compte de tous ces facteurs, (sociologiques autant qu'économiques) que la planification du développement pourra prendre son sens véritable.

Gérald Fortin

Standards of Wage Determination, by Paul Bullock, Institute of Industrial Relations, University of California, Los Angeles, 1960, 99 pp. \$0.75.

Il s'agit d'une brochure intéressante dont le but est d'analyser l'importance de certaines procédures, techniques et méthodes utilisées dans la fixation des salaires. L'auteur, Paul Bullock, étudie les critères suivants considérés lors de la négociation: comparaisons avec d'autres entreprises ou l'industrie en général, coût de la vie, capacité de payer, budget familial, pouvoir d'achat, facteurs techniques et autres.

Les comparaisons permettent aux travailleurs moins bien rémunérés de viser à l'obtention de salaires comparables à ceux qui sont les mieux rémunérés.

Le coût de la vie est un critère populaire en période d'inflation, mais oublié du travailleur, et pour cause, en période de régression.

La capacité de payer, quoique n'étant pas un facteur explicite dans les négociations, joue un rôle important. La résistance à l'augmentation des salaires sera faible si le marché est fort et sera fort si les affaires ralentissent.

La productivité est souvent invoquée mais a peu d'influence car elle est difficile d'interprétation et les négociateurs se perdent vite dans les masses de données statistiques.

Le budget familial a lui aussi peu d'influence car il est difficile de préciser un standard adéquat et de le traduire en terme de cents par heure. 
L'auteur continue ainsi pour les autres critères énumérés plus haut. Dans sa conclusion, il souligne que les gages sont beaucoup plus qu'un taux horaire, et qu'un groupe de facteurs complexes détermine l'employé à accomplir son travail. Ses gages sont composés de bénéfices tangibles et intangibles et ses réactions, vis-à-vis les changements dans ces bénéfices, sont imprévisibles.

JeAn-Paul DEschênes

Wage Determination, An Analysis of Wage Criteria, by Jules Backman, D. Van Nostrand Company, Inc., N.J., 1959, 316 pp.

Voilà un livre qui plaira à ceux qui sont intéressés à la détermination des taux de rémunération, sans être particulièrement intéressés à la théorie des salaires, et à ceux surtout qui ont l'occasion de participer à l'élaboration de ces taux au cours de négociations ou d'arbitrage. Le livre, en effet, ne peut rebuter ceux dont l'esprit répugne à l'analyse de choses abstraites, puisqu'on n'y traite aucunement de la théorie des salaires. Celle-là, dit l'auteur, ne sert à rien dans les négociations collectives. Ce dernier se propose donc de ne passer en revue que les principaux critères utilisés par les parties en présence pour accepter ou refuser la prétention de l'autre: Comparaison des salaires, budgets familiaux, coût de la vie, productivité, capacité de payer et contexte économique général. L'intérêt de cette revue est triple: on y trouve pour chacun des critères les différentes façons dont on peut les utiliser, les limites à chacune de ces façons et une référence à leurs sources statistiques.

Les références aux sources statistiques sont, pour la plupart, américaines. Pour cette raison, elles ne sont pas d'une très grande utilité pour les négociateurs canadiens. L'auteur cependant, à diverses occasions, complète son exposé par des données générales sur la situation canadienne.

Le livre dans l'ensemble apporte une contribution valable aux éclaircissements qu'il est nécessaire d'apporter afin que soient utilisés avec plus de correction les critères habituels de détermination des salaires. Pour le lecteur non averti, le livre comporte cependant certaines trappes.
Tout d'abord, l'image des restrictions à l'utilisation de certains critères ou celle de leurs implications n'est pas toujours complète. Je n'en veux citer qu'un exemple. A l'occasion de la discussion sur le critère du coût de la vie, nulle mention n'est faite du délai habituellement courant entre l'augmentation du coût de la vie et l'augmentation des salaires. Ce délai a pour conséquence de ne jamais permettre au travailleur la possibilité de maintenir son standard de vie.

Ensuite, l'analyse est parfois fautive et seul un esprit averti peut en déceler les faiblesses. C'est ainsi que l'affirmation suivante (p. 238) malgré sa sûreté apparente n'a rien de très sûr: $\ll . .$. the increase in the total employment costs in the steel industry between 1945 et 1955 was greater than total profits before taxes in 1955. In other words, if steel prices had not been increased, the industry would have operated at a substantial loss in 1955 s. En effet le maintien des profits - malgré l'augmentation du coût total de l'emploi - peut se réaliser si l'augmentation des quantités produites est plus que proportionnelle à l'augmentation des coûts de l'emploi ou si des changements dans d'autres facteurs de production ont conduit à des économies. Des faits ci-haut mentionnés, il ne s'ensuit pas nécessairement qu'il y a eu augmentation des prix.

Enfin, il nous a semblé que l'auteur. malgré un effort réel et apparent pour présenter le tout de façon objective, n'y réussit pas toujours. On peut déceler dans son texte des sympathies pour l'une des deux parties en cause lors de la détermination des taux de rémunération. Elle se traduit dans le fait que ce qui peut $y$ avoir d'incomplet ou d'incorrect dans son étude est habituellement au désavantage de celui qui offre son travail.

Malgré ces restrictions - d'une certaine importance - il reste quand même que l'auteur apporte clairement une contribution utile à ceux dont c'est le devoir d'éprouver les limites et implications des critères de détermination du salaire.

Jacques St-Laufent

« Automation and the Worker: a Study of Social Change in Power Plants s, by Floyd C. Mann and L. Richard Hoffman, Henry Holt and Co., New York, 1960,272 pp. plus XIV, $\$ 4.50$ 